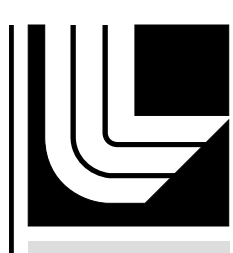

LA W RENCE LIVERM ORE N A TIO NAL LABORATORY

Ridge-Valley Graphs: Combinatorial Ridge Detection Using Jacobi Sets

G. Norgard, P. T. Bremer

February 10, 2011 
This document was prepared as an account of work sponsored by an agency of the United States government. Neither the United States government nor Lawrence Livermore National Security, LLC, nor any of their employees makes any warranty, expressed or implied, or assumes any legal liability or responsibility for the accuracy, completeness, or usefulness of any information, apparatus, product, or process disclosed, or represents that its use would not infringe privately owned rights. Reference herein to any specific commercial product, process, or service by trade name, trademark, manufacturer, or otherwise does not necessarily constitute or imply its endorsement, recommendation, or favoring by the United States government or Lawrence Livermore National Security, LLC. The views and opinions of authors expressed herein do not necessarily state or reflect those of the United States government or Lawrence Livermore National Security, LLC, and shall not be used for advertising or product endorsement purposes.

This work performed under the auspices of the U.S. Department of Energy by Lawrence Livermore National Laboratory under Contract DE-AC52-07NA27344. 


\title{
Ridge-Valley Graphs: Combinatorial Ridge Detection Using Jacobi Sets
}

\author{
Greg Norgard ${ }^{\mathrm{a}}$, Peer-Timo Bremer ${ }^{\mathrm{b}}$ \\ ${ }^{a}$ Numerica Corporation, 4850 Hahns Peak Drive, Suite 200, Loveland, CO 80538 \\ ${ }^{b}$ Lawrence Livermore National Laboratory, 7000 East Avenue, L-422, Livermore CA 94551
}

\begin{abstract}
Ridges are one of the key feature of interest in areas such as computer vision and image processing. Even though a significant amount of research has been directed to defining and extracting ridges some fundamental challenges remain. For example, the most popular ridge definition (height ridge) is not invariant under monotonic transformations and its global structure is typically ignored during numerical computations. Furthermore, many existing algorithm are based on numerical heuristics and are rarely guaranteed to produce consistent results. In this paper a small change to the height ridge definition gives a definition that is consistent with the desired invariants. Nevertheless, we show that this definition results in similar structures compared to the most common traditional approach and that both formulations are equivalent for quadratic functions. Furthermore, this definition can be cast in the form of a degenerate Jacobi set, which allows insights into the global structure of ridges. In particular, we introduce the Ridge-Valley graph as the complete description of all ridges in an image. Analyzing this graph reveals similar invariants to those found for traditional ridge formulations, such as the fact that ridges do not merge. Finally, using the connection to Jacobi sets we describe a new combinatorial algorithm to extract the Ridge-Valley graph from sampled images that is guaranteed to produce a valid structure.
\end{abstract}

\section{Introduction}

Ridges, often described intuitively as the crests connecting mountain peaks, are some of the most sought after features in areas ranging from computer vision $[1,2]$ and image processing [3] to tensor analysis [4, 5] and combustion simulations [6]. Consequently, defining and extracting ridges from digital data

This work was performed under the auspices of the U.S. Department of Energy by Lawrence Livermore National Laboratory under Contract DE-AC52-07NA27344.UCRL LLNL-TR-470098

Email addresses: gregnorgard@gmail.com (Greg Norgard), ptbremer@acm.org (Peer-Timo Bremer) 
has received significant attention across different communities resulting in various competing concepts and a plethora of algorithms. Here, we are concerned with ridge extraction from two-dimensional images, one of the oldest problems in data analysis. Nevertheless, despite significant efforts, fundamental theoretical as well as practical problems remain. On the theoretical side, a set of five characteristics desirable in ridges are defined [3], yet the most commonly used definition violates one of them. Furthermore, one of these characteristics is the desire for an entirely local definition and traditional algorithms typically disregard the expected global structure of ridges. However, without a clear understanding of the desired outcome evaluating and validating algorithms that extract ridges remains subjective and ad-hoc. To address these shortcomings additional algorithms and heuristics have been developed to filter and postprocess the results of the initial extraction. Again, without knowledge of the expected outcome designing such frameworks is challenging.

In this paper we revisit an often neglected definition of ridge, which we will refer to as Jacobi ridges, that observes all five desired invariants. This definition is subtly different from the common height ridge definition, with the resulting ridges nearly identical and can be shown to be equivalent for quadratic functions. Furthermore, the definition can be expressed in form of a Jacobi set of a function and its gradient magnitude, which provides insight into the global structure of these ridges. The known properties of Jacobi sets for generic functions combined with an in depth analysis of the degeneracies in the non-generic case allow us to describe the Ridge-Valley graph, a global representation of all ridges. The Ridge-Valley graph reveals necessary invariants that can be used to guide and validate ridge extraction algorithms. For example, analyzing the graph makes clear that ridges cannot merge or split and that there exist sibling structures called pseudo-ridges that when combined with ridges form closed non-intersecting loops. Finally, the connection to Jacobi sets allows us to define an equivalent concept of ridges for piecewise linear functions and a new combinatorial algorithm to extract Ridge-Valley graphs. The resulting framework is easy to compute and is numerically more robust, due to its combinatorial nature. Our algorithm, for the first time, guarantees the extraction of a valid ridge structure equivalent to that of a smooth function. Ultimately, this paper introduces a new theoretical framework to understand, analyze, and extract ridges of piece-wise linear functions using an abstract graph encoding of the global structure of ridges. Similar to the Morse-Smale complex for topological information, the Ridge-Valley graph promises a deeper understanding of the nature of the examined ridges and more effective algorithms to extract them.

\subsection{Related Work}

The study of ridge-lines actually begins with the examination of valley-lines discussed in a paper by De Saint-Venant in 1852 [7] describing "courses" (valleys). De Saint-Venant's definition, when followed to its furthest extent are the Jacobi ridges, that this paper examines. Since then, numerous authors have addressed the issue often in confusing and contradictory ways. This historical body of work includes Boussinesq [8, 9], Breton de Champ[10], Jordan [11] 
and Rothe [12] and we encourage the reader to review Koenderink and van Doorn's [13] excellent overview.

The modern day results of these efforts are multiple ridge definitions that, while related, are not equivalent. Eberly et al. [3] discuss some of the more notable variants in great detail and also lays out a locality property and four general invariants that an ideal ridge definition should fulfill, see Section 2. In modern applications, the height ridge definition introduced by Haralick [14] and extended by Eberly et al. [3] has become the most prevalent. It is used in a large number of applications in a variety of areas despite not satisfying one of the desired properties. Furthermore, in a rarely cited paper, Damon [15] introduces ridge-valley-connector-curves which describe the global structure of height ridges similar to our Ridge-Valley graph for Jacobi ridges.

Based on this theory, there exists an extensive collection of algorithms to extract (height) ridges and even a cursory review is beyond the scope of this paper. For a detailed discussion on some of the more popular approaches, we refer the reader to $[16,2,17,18,19]$. It has been previously noted that all of the ridges and valleys in the height ridge definition, must be points where the gradient of the height function and the gradient of the magnitude are aligned $[17,20,19,13]$, but the subtle consequences of this observation have not been examined, which is one of the results of this paper. In their recent paper, Sadlo and Peikert [19] take advantage of this fact to extract "raw features," which are then filtered to produce ridges and valleys. These "raw features" with their "contour distance filter" are the same ridge/valley definition presented in this paper. In their paper, they extract these features by doing level set extraction on a scalar field derived from the height field. Numerical level set extraction will produce 1-dimensional loops, however we show in the course of this paper, that these features have a more complex structure and will in fact cross at every critical point. Thus without some sort of heuristic or connecting procedure the ridges in [19] will be disconnected at every critical point, which they admit is a difficulty. The algorithm presented in this paper avoids this shortcoming by employing a combinatorial algorithm, which is guaranteed to connect ridges and valleys through critical points.

In practice, a low pass filter is often applied during the extraction of ridges. While the primary effect is the extraction ridges of the appropriate length of scale, these filters will also change the geometric location of such structures and may alter the results. Furthermore, a shortcoming of eigenvector-based approaches is that an eigenvector has no definitive orientation and reconstructing the ordering of eigenvalues and associated eigenvectors can be problematic. Additionally, the numerical errors involved in approximating eigenvalues and eigenvectors can lead to disconnected ridges and additional artifacts. Thus, a large amount of effort is spent on attempts to correct the structure through various filters, thresholds, and application dependent heuristics.

Instead, we will show that Jacobi ridges can be expressed as non-generic Jacobi sets, which implies a rigorous global structure akin to the connector curves. Jacobi sets have been introduced by Edelsbrunner and Harer [21] as the set of points at which the gradients of two (or more) functions are aligned. Further- 
more, they introduced a simple combinatorial algorithm to extract Jacobi sets for piecewise linear data. Currently Jacobi sets have found usage in different areas of analysis. Mascarenhas et al. [22] showed how Jacobi sets can be used to track critical points in time-dependent simulations and Natarajan et al. [23] derived a new similarity metric based on Jacobi sets. Luo et al. [24] extract an approximate Jacobi set from point cloud data to extract features from molecular surfaces as well as contours of geometric models. In this paper, we utilize a recent extension to the original algorithm [25] which considers a previously ignored property of the Jacobi set and in our application produces qualitatively better results. Furthermore, we demonstrate how to adapt the algorithm to handle non-generic Jacobi sets, in order to enforce the global structure of ridge lines. Our contributions in detail are:

1. An examination of the Jacobi ridge definition which fulfills all desired invariants and rigorous comparisons to the height ridge definition;

2. A formulation of Jacobi ridges in the form of a non-generic Jacobi set;

3. Introduction of the Ridge-Valley graph, a new structure describing the global structure of Jacobi ridges; and

4. A new combinatorial algorithm to extract Jacobi sets for two non-generic functions guaranteed to produce the correct structure.

\section{Background}

In this paper we are concerned with ridge- and valley-lines of a two-dimensional function $f$. For simplicity of the presentation we will assume $f: \mathbb{R}^{2} \rightarrow \mathbb{R}$, but the results could be extended to more general two-dimensional manifolds. As will become apparent, the definition of ridges and valleys is symmetric and for brevity we will restrict much of the discussion to the case of ridges, but all results equally apply to valleys. Throughout the paper we use $\nabla f$ to denote the gradient of $f, H$ to denote the Hessian matrix of $f$, and $R$ to denote the rotation operator, that will rotate a vector $90^{\circ}$ in the counter-clockwise direction.

In their seminal paper, Eberly et al. [3] compare several definitions of ridges, extend Haralick's "height" definition [14] into multiple dimensions, and establish a set of desired invariants for ridge definitions. Their conclusion is that the so-called height ridges produce qualitatively superior results. This sentiment appears to be wide spread since height ridges are a commonly used ridge structure $[3,18,26,20,27]$. However, Eberly et al. also note that this particular definition does not satisfy invariance under monotonic transformations, while other definitions satisfy all the desired invariants. In this section, we review the set of invariants and the definition of height ridges, and extend the classification by introducing pseudo-ridges/-valleys.

According to [3] a ridge of $f$ should be:

1. defined locally, depending only on information in an $\epsilon$-neighborhood around a given point;

2. invariant under translations in the spatial variables; 
3. invariant under rotations in the spatial variables;

4. invariant under uniform magnification in the spatial variables; and

5. invariant under monotonic transformations of $f$.

All of these invariants are of practical importance since they are required for a localized algorithm invariant to spatial transformations as well as invariant to common transformations of $f$ such as, for example, a logarithmic scaling.

The definition of a height ridge uses the directions of highest and lowest curvature (which are the eigenvectors of $H$ ) and their relation to the gradient. To streamline the discussion in Section 3.1 we first define a set of height points that are subsequently classified further:

Definition 2.1 (Height Point). A point $x \in \mathbb{R}^{2}$ is called $a$ height point if $\nabla f(x) \neq 0, \lambda_{1}, \lambda_{2} \neq 0, \lambda_{1} \neq \lambda_{2}$ eigenvalues of $H(x)$, and $H(x) \nabla f(x)=\lambda \nabla f(x)$ for some $\lambda \in \mathbb{R}$.

Intuitively, the set of height points are the points in $\mathbb{R}^{2}$ where the gradient is aligned with one of the directions of principal curvature of the graph of $f$. By excluding points with a zero gradient and points with an eigenvalue of zero or a higher multiplicity eigenvalue, the set of height points is an open set with these special points as boundary. Ridges and valleys are certain subsets of height points:

Definition 2.2 (Height Ridges/Valleys). Let $x \in \mathbb{R}^{2}$ be a height point and $\lambda_{1}<\lambda_{2}$ be the eigenvalues of $H(x)$. If $H(x) \nabla f(x)=\lambda_{2} \nabla f(x)$ then $x$ is part of $a$ height ridge for $\lambda_{1}<0$ and part of a height pseudo-valley for $\lambda_{1}>0$. Symmetrically, if $H(x) \nabla f(x)=\lambda_{1} \nabla f(x)$ and $\lambda_{2}>0$ then $x$ is on a height valley and on a height pseudo-ridge for $\lambda_{2}<0$, see Table 1 .

\begin{tabular}{|l|c|c|}
\hline Height Classification & Alig. of $\nabla f$ & Eig.values of $H$ \\
\hline ridge point & $H \nabla f=\lambda_{2} \nabla f$ & $\lambda_{1}<0$ \\
valley point & $H \nabla f=\lambda_{1} \nabla f$ & $\lambda_{2}>0$ \\
pseudo-ridge point & $H \nabla f=\lambda_{1} \nabla f$ & $\lambda_{2}<0$ \\
pseudo-valley point & $H \nabla f=\lambda_{2} \nabla f$ & $\lambda_{1}>0$ \\
\hline
\end{tabular}

Table 1: The classification of a height point according to the eigenvalues $\lambda_{1}<\lambda_{2}$ of $H$.

Intuitively, a point is classified as height (pseudo-)ridge if $f$ has a local maximum in the direction orthogonal to the gradient. Furthermore, if the gradient is aligned with the direction of maximal curvature $x$ is called a ridge otherwise $x$ is called a pseudo-ridge. Symmetrically, (pseudo-)valleys are points at which $f$ is locally minimal orthogonal to the gradient and valleys have the gradient aligned with the direction of minimal curvature ${ }^{1}$.

In practice, one is typically only interested in either the ridges or the valleys. However, as will be discussed in Section 4, pseudo-ridges/valleys play an important role in understanding the global structure of ridges/valleys in general.

${ }^{1}$ These form the ridges, valleys, $\mathrm{r}-$, and v-connectors of [15] 
While the height ridge definition closely follows human intuition and satisfies properties 1-4, it is not invariant under monotonic transformations of $f$. As in [3], consider the example $f=1-x^{2}-2 y^{2}$ and the monotonic transformation $h=\ln (1+t)$ : The point $(1,0)$ is part of a height ridge of $f$, but not of $h \circ f$.

\section{Jacobi Ridges}

As discussed above, height ridges are a commonly used definition even though they are not invariant under monotonic transformations. This section introduces a new formulation of ridges and valleys closely related to one originally presented by De Saint-Venant in 1852 [7]. Subsequently, we demonstrate that the new definition observes all five characteristics, yet is (perhaps surprisingly) close to the height ridge definition. In the following discussion, we will use $g=\|\nabla f\|^{2}$ to indicate the squared gradient magnitude of $f$ and define $L(x)=f^{-1}(f(x))$ as the level set of $f$ incident to $x$. As before, we first define a set of candidate points:

Definition 3.1 (Jacobi Points). A point $x \in \mathbb{R}^{2}$ is called a Jacobi point if $\nabla f(x) \neq 0$ and for $\vec{t}=R \nabla f$ (the vector tangent to $L(x)$ ) and $\gamma$ a parametrization of $L(x)$ we have: $\frac{\partial^{2} f}{\partial \vec{t}^{2}} \neq 0$ and $\frac{\partial}{\partial s} g(\gamma)=0$ and $\frac{\partial^{2}}{\partial s^{2}} g(\gamma) \neq 0$.

Intuitively, Jacobi points are points where the gradient magnitude restricted to a level set of $f$ is either maximal or minimal. Note that, $\frac{\partial f}{\partial \vec{t}}=0$ is true by definition and $\frac{\partial^{2} f}{\partial \vec{t}^{2}} \neq 0$ removes the points with zero level set curvature. Again the set of Jacobi points forms an open set whose boundary are points with zero gradient, points with zero level set curvature, and the inflection points of $g$ restricted to level sets of $f$. Jacobi points are classified further according to the behavior of $f$ tangentially to its level sets:

Definition 3.2 (Jacobi Ridges/Valleys). Let $x$ be a Jacobi point of $f$ then $x$ is part of a Jacobi pseudo-ridge (ridge) if $g$ is maximal (minimal) along $L(x)$ and $f$ is maximal tangent to $L(x)$. Symmetrically, $x$ is part of a pseudo-valley (valley) if $g$ is maximal (minimal) along $L(x)$ and $f$ is minimal tangent to $L(x)$.

\begin{tabular}{|l|c|c|}
\hline Jacobi Classification & Along $L(x)$ & Tangent to $L(x)$ \\
\hline ridge point & $\min$ of $g$ & $\max$ of $f$ \\
valley point & $\min$ of $g$ & $\min$ of $f$ \\
pseudo-ridge point & $\max$ of $g$ & $\max$ of $f$ \\
pseudo-valley point & $\max$ of $g$ & $\min$ of $f$ \\
\hline
\end{tabular}

Table 2: The classification of a Jacobi point $x \in \mathbb{R}^{2}$ according to the behavior of $g$ along level sets and $f$ tangentially to the level sets.

The intuition behind Jacobi ridges is based on walking around a mountain top along a level set. One encounters points where the terrain is least/most steep, which are the points along ridges/pseudo-ridges. 
An important fact to observe is that Jacobi ridges satisfy all five of the desirable properties: Clearly, the definition is local and invariant under spatial translations and rotations. Spatial magnification is equivalent to uniformly scaling the gradient, which does not affect the existences or classification of extrema of $g$ along level sets of $f$. Finally, we can prove the following lemma:

Lemma 3.3. Jacobi ridge points and their classifications are invariant under monotonic transformations of $f$.

Proof Let $h: \mathbb{R} \rightarrow \mathbb{R}$ be a monotonic transformation with $h^{\prime}>0$ and $\phi=h \circ f$ be the transformed $f$. Then $\nabla \phi(x)=h^{\prime}(f(x)) \nabla f(x)$. For each level set $L_{f}$ of $f$ at value $a$ there exists an equivalent level set $L_{\phi}$ of $\phi$ at $h(a)$ such that $L_{f}=L_{\phi}$. By definition, $f$ is constant along $L_{f}, h^{\prime}(f(x))$ is constant and thus for $c=h^{\prime}(f(x))$ we have that $\|\nabla \phi\|^{2}=c^{2}\|\nabla f\|^{2}$ along $L_{f}$. Since, multiplying $\|\nabla f\|^{2}$ with a constant factor will not change the location of extrema, Jacobi points and classifications are invariant under monotonic transformations.

\subsection{Comparison}

Given that the two definitions are established using different intuitive criteria and the fact that, unlike height ridges, Jacobi ridges are invariant under monotonic transformations, one would expect the two ridge definitions to be quite different. However, they are more similar than one might expect. The following lemma shows that the points identified by both definitions are generally the same.

Lemma 3.4. Let $C_{\text {height }}$ and $C_{J a c o b i}$ be the closure of all height and Jacobi points respectively. Then,

$$
C_{\text {height }}=C_{\text {Jacobi }}=\left\{x \in \mathbb{R}^{2} \mid(\nabla f(x))^{T} H(x)(R \nabla f(x))=0\right\} .
$$

Proof For height points this is true by construction.

Clearly, the critical points of $f$ are in $C_{J a c o b i}$ and in $\left\{x \in \mathbb{R}^{2} \mid(\nabla f(x))^{T} H(x)(R \nabla f(x))=\right.$ $0\}$. For the remaining Jacobi points we have $\frac{\partial}{\partial s} g(\gamma)=0$. Let $\gamma(s)$ be a parametrization of the level set of $f$ such that $\frac{\partial}{\partial s} \gamma=R \nabla f$. Differentiating $g$ with respect to $s$ results in

$$
\frac{\partial}{\partial s} g(\gamma)=\frac{\partial}{\partial s}\|\nabla f(\gamma)\|^{2}=\frac{\partial}{\partial s}\left(\nabla f^{T} \nabla f\right)=2 \nabla f^{T} H(f) \gamma^{\prime}(s)=2(\nabla f)^{T} H(f)(R \nabla f)
$$

which proves the lemma.

Lemma 3.4 shows that the two definitions result in the same set of points up to closure. It also shows that when the magnitude of the gradient is maximized or minimized on a level set of $f$, then the gradient is an eigenvector of the Hessian. However, it makes no guarantees about the ordering of the eigenvalues of the Hessian. This is the primary difference between the two definitions. The distinction between a pseudo and non-pseudo point in the height definition 
depends on the ordering of the eigenvalues, while the Jacobi definition distinguishes based on whether the gradient magnitude is maximized or minimized. Since, a monotonic transformation can change the ordering of the eigenvalues of a Hessian it can change the classification of the height points, explaining why the Jacobi ridge definition is invariant under monotonic transformations, while the height definition is not.

In both definitions, the determination of the ridge/valley property is identical. At a height or Jacobi point the vector tangent to the level set is an eigenvector of the Hessian, thus the sign of the eigenvalue determines if that point is a maximum or minimum of $f$ tangent to the level set. We also note that the sign of the level set curvature defined as

$$
L S C=-\nabla \cdot \frac{\nabla f}{\|\nabla f\|}=-\frac{(R \nabla f)^{T} H R \nabla f}{\|\nabla f\|^{3}}=\frac{\lambda_{R \nabla f}}{\|\nabla f\|}
$$

will also determine whether a point is ridge or valley.

Thus we find that the only difference in the two definitions relies on the classification of the points that satisfy the equation $(\nabla f)^{T} H(f)(R \nabla f)=0$. Furthermore, we find that the two definitions will "often" agree in the classification. The following lemma establishes that for quadratic functions the two definitions are equivalent.

Lemma 3.5. Let $x$ be a height point and let $f$ be locally quadratic at $x \in \mathbb{R}$. The point $x$ has the same classification using both the Jacobi and height ridge definition.

Proof Let $\gamma(s)$ be a parametrization of $g$ along a level set of $f$ such that $\frac{\partial}{\partial s} \gamma=R \nabla f$. As derived above: $\frac{\partial}{\partial s} g=2(\nabla f)^{T} H(R \nabla f)$. Consulting with mathematical software, the second derivative can be expressed as

$$
\frac{\partial^{2}}{\partial s^{2}} g=-2 \operatorname{det}(H(f))+2 \frac{\left((R \nabla f)^{T} H(f)(R \nabla f)\right)^{2}}{\|\nabla f\|^{4}}+\frac{(R \nabla f)(\nabla(H(f)) \nabla f)(R \nabla f)}{\|\nabla f\|^{2}} .
$$

As $\nabla(H(f))$ is a tensor of third order derivatives and $f$ is assumed to be quadratic, we find $\nabla(H(f))=0$. Since, $x$ is a height point, the eigenvectors of $H(f)$ are aligned with the gradient and the vector tangent to the level set. Let $\lambda_{t}$ and $\lambda_{g}$ be the eigenvalues of $H(f)$ such that $H(f)(\nabla f)=\lambda_{g} \nabla f$ and $H(f)(R \nabla f)=\lambda_{t} \nabla f$. It follows that

$$
\frac{\partial^{2}}{\partial s^{2}} g=-2 \lambda_{t} \lambda_{g}+2 \lambda_{t}^{2}=2 \lambda_{t}\left(\lambda_{t}-\lambda_{g}\right)
$$

The sign of $\frac{\partial^{2}}{\partial s^{2}} g$ and $\lambda_{t}$ will determine the classification using the Jacobi ridge definition. Additionally, through equation 3 they imply an ordering on the eigenvalues $\lambda_{t, g}$. This ordering will determine the classification using the height ridge definition. Using tables 1 and 2, the classifications are seen to be equivalent. 
From this lemma, it is clear that the differences between the two definitions of ridges resides in the third derivatives. Thus, when the behavior of the function $f$ is dominated by second and lower order effects, one would expect the two definitions to produce similar results. In practice, Sadlo and Peikert [19] found that the Jacobi ridges were slightly, qualitatively preferable.

Additionally, we note that the differences in classifications also effects how the classifications transition along the height/Jacobi points. Since $f$ is smooth the classification cannot change within connected subsets. Consider the height definition: A sequence of ridge/valley points can transition to a sequence of pseudo-ridge/valley points or vice versa by passing through a point where the eigenvalues of $H$ are equal. The same transition can occur in the Jacobi definition, but at points where $\frac{\partial}{\partial s} g=0$. From Equations 2 and 3 we can see that these points are not generally the same, with a notable exception of when the third derivatives are zero. In Figure 1(a) we see an example of these transition points for the Jacobi ridge definition.

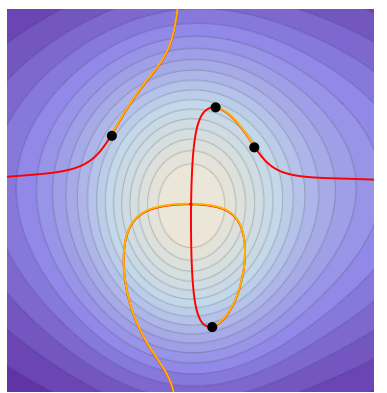

(a)

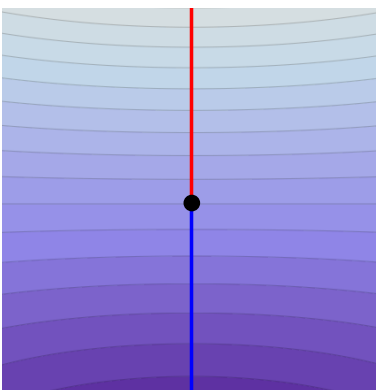

(b)

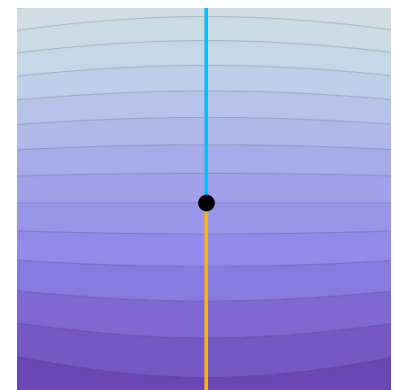

(c)

Figure 1: (a) An example of the transition of (pseudo-)ridge/valley points. The contours of $f=\exp \left[-(x-0.1)^{2}-2(y-0.1)^{2}\right]+\exp \left[-3\left(2 x^{2}+y^{2}\right)\right]$ are displayed along with with the level set $(\nabla f)^{T} H(f)(R \nabla f)=0$. Red lines represent Jacobi ridges and the orange lines pseudo-ridges. Inflection points of $g$ along the level sets of $f$ are marked as black dots. (b) A ridge (red line) of $f=-y x^{2}+5 y+y^{2}$ is shown transitioning to a valley (blue line) at a point where the level set curvature is zero. (c) A pseudo-valley (teal line) of $f=-y x^{2}+5 y+y^{2}$ is shown transitioning to a pseudo-ridge (orange line) at a point where the level set curvature is zero.

Additionally when height/Jacobi points run through a point with a level set curvature of 0 (the eigenvalue of the tangent eigenvector is 0 ), they can change classification. In this case, however, there are significant differences. In the height definition, points will generically transition from ridge to pseudo-valley, or from valley to pseudo-ridge. In the Jacobi definition points transition from (pseudo-)ridge to (pseudo-)valley. An example of such a transition is shown in Figure 1, where the Jacobi classification is displayed. Table 3 briefly describes how and when the classification can change. We note that, generically, the height definition does not allow a (pseudo-)ridge to (pseudo-)valley transition and that the Jacobi definition does not allow a ridge/valley to pseudo-valley/pseudo-ridge transition. For a more complete description of height ridge structure we refer the reader to [15]. 


\begin{tabular}{|l|c|c|}
\hline Transition & Height & Jacobi \\
\hline $\begin{array}{l}\text { ridge } \leftrightarrow \text { pseudo-ridge } \\
\text { valley } \leftrightarrow \text { pseudo-valley }\end{array}$ & $\lambda_{1}=\lambda_{2}$ & $\frac{\partial}{\partial s} g=0$ \\
\hline $\begin{array}{l}\text { ridge } \leftrightarrow \text { pseudo-valley } \\
\text { valley } \leftrightarrow \text { pseudo-ridge }\end{array}$ & $\lambda_{1}$ or $\lambda_{2}=0$ & - \\
\hline $\begin{array}{l}\text { ridge } \leftrightarrow \text { valley } \\
\text { pseudo-ridge } \leftrightarrow \text { pseudo-valley }\end{array}$ & - & $\lambda_{1}$ or $\lambda_{2}=0$ \\
\hline
\end{tabular}

Table 3: A description on how the classification of height points and Jacobi points can transition. In both cases the pseudo/non-pseudo classification can switch, but under different conditions. For height points the eigenvalues must be equal, and for Jacobi points $g$ must have an inflection point on a level set of $f$. At points where an eigenvalue is zero, either definition can switch classification, however the type of switch is different.

By comparing the two similar, yet subtly different definitions, we note that the Jacobi definition has significant advantages both theoretically and practically. Theoretically, Jacobi ridges satisfies all of the desired characteristics defined by Eberly. Additionally the formulation as extrema of a function restricted to the level sets of a second function allows us to cast Jacobi ridges as non-generic Jacobi sets, see below, providing further insights. Practically, we will show in Section 5, that the geometry of Jacobi ridges can be computed using a combinatorial extraction algorithm, bypassing any need for a potentially unstable eigenvector/eigenvalue computation and guaranteeing a correct global structure.

\section{Ridge-Valley Graph}

In this section we will show how the Jacobi definition of ridges can be expressed as a non-generic Jacobi set and use the resulting insights to define the Ridge-Valley graph, encoding the global structure of ridges and valleys. We first briefly introduce Jacobi sets for two functions on a two-dimensional manifold $\mathbb{M}$ and refer the reader to [21] for a more in depth discussion.

A point of $f$ is called critical if the gradient at that point is zero. A critical point $p$ of $f$ is called degenerate if its Hessian matrix is singular. Furthermore, $f(p)$ is called a critical value of $f$, with a regular value referring to a noncritical value. A smooth function is called Morse if all its critical points are non-degenerate and have pair-wise distinct function values. Finally, two Morse functions $f$ and $g$ are called generic if they do not share critical points. We are interested in two Morse functions $f$ and $g$ and in particular in the restrictions of $g$ to level sets of $f$. For a regular value $t \in \mathbb{R}$ the level set $f^{-1}(t)$ is a smooth 1-manifold and the restriction of $g$ to this level set is a smooth function $g_{t}$. We can now define the Jacobi set of two Morse functions as:

Definition 4.1. The Jacobi set $\mathbb{J}(f, g)$ is the closure of the set of critical points of $g_{t}$ :

$$
\mathbb{J}(f, g)=\operatorname{cl}\left\{x \in \mathbb{M} \mid x \text { is critical point of } g_{t}\right\},
$$

for some regular value $t \in R$. 
The closure operations adds the critical points of $g$ restricted to the level sets at critical values $t$ as well as critical points of $f$, which form singularities in these level sets. The definition is symmetric $\mathbb{J}(f, g)=\mathbb{J}(g, f)$ and results in a set of smoothly embedded 1-manifolds, see [21].

Theorem 4.2 (SMOOTH EMBEDDING THEOREM). The Jacobi set of two generic Morse functions $f, g: \mathbb{M} \rightarrow \mathbb{R}$ is a smoothly embedded 1-manifold in $\mathbb{M}$

Considering Definitions 3.1 and 4.1 it is easy to see that the closure of all Jacobi points is the Jacobi set $\mathbb{J}(f, g)$ of $f$ and $g=\|\nabla f\|^{2}$. Since all critical points of $f$ are minima of $g$ the two functions are not generic and to emphasize this fact we will call their Jacobi set non-generic. The main consequence of the shared critical points is that at critical points of $f$ the smooth embedding theorem does not hold.

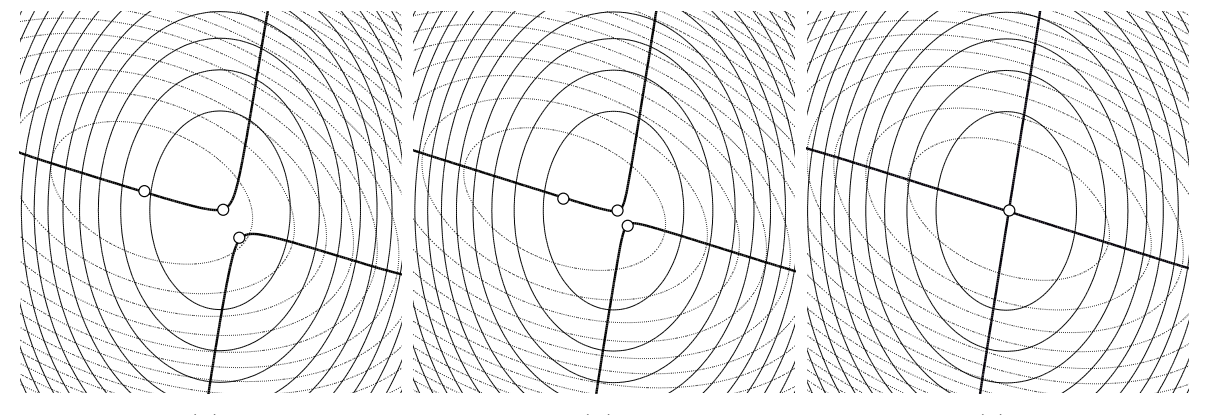

(a)

(b)

(c)

Figure 2: Here the contours of two quadratic minima $f$ and $g$ are shown in light and dark lines. The Jacobi set is shown as the thick line, with the critical points and birth-death points shown as circles. As the two minima approach each other, the birth-death point approaches the minima. When the minima become co-located, the birth-death point also become co-located giving a valence four point.

To analyze the Jacobi ridge structure in the neighborhood of critical points of $f$ it is helpful to study the effects of two critical points of two functions approaching each other. Figure 2 shows the level sets of two quadratic minima and their Jacobi set with the two sets of contours represented in light and dark and the Jacobi set as a bold line. The Jacobi points are shown as lines with their boundary points indicated by circles. In Figure 2(a) the top component of the Jacobi set contains the minima of both $f$ and $g$ while the bottom component contains an inflection point of $g$ restricted to level sets of $f$, also called a birthdeath point. As the two minima approach each other, see Figure 2(b), the birth-death point becomes closer as well. Finally, in the non-generic situation of a shared critical point both minima and the birth-death point are co-located and create a valence four node in the Jacobi set, see Figure 2(c). We formalize this result for the Jacobi set of $f$ and $\|\nabla f\|^{2}$ with the following theorem. 
Theorem 4.3. Let $f$ be a smooth function such that at all critical points of $f$ the Hessian have distinct, non-zero eigenvalues. Then the Jacobi set $\mathbb{J}\left(f,\|\nabla f\|^{2}\right)$ will have valence four at all critical points of $f$.

Proof Let $g=\|\nabla f\|^{2}$ then $\mathbb{J}(f, g)$ is also defined as the 0 level set of $(\nabla g)^{T}(R \nabla f)$. We will prove the theorem by showing that at critical points of $f$ this function has a saddle point with function value 0 and a non-degenerate Hessian.

Wlg. let $f$ have a critical point at the origin with eigenvectors of the Hessian, $H$, aligned with the coordinate axes. Since $f$ is Morse, it can be approximated by a quadratic near a critical point of $f$. Thus near the origin $f \approx a x^{2}+b y^{2}$. For simplicity assume that $f$ is equal to its quadratic approximation $f=a x^{2}+b y^{2}$. This assumption is allowable as the higher order terms in the approximation do not affect the result. With a few minor calculations

$$
\begin{array}{rlr}
\nabla f & = & \langle 2 a x, 2 b y\rangle, \\
g=\|\nabla f\|^{2} & = & 4 a^{2} x^{2}+4 b^{2} y^{2}, \\
\nabla g & = & \left\langle 8 a^{2} x, 8 b^{2} y\right\rangle, \\
R \nabla f & = & \langle-2 b y, 2 a x\rangle, \\
(\nabla g)^{T}(R \nabla f) & = & \left(-16 a^{2} b+16 a b^{2}\right) x y,
\end{array}
$$

we can see that $(\nabla g)^{T}(R \nabla f)$ will indeed have a saddle of function value 0 at the origin. As $a$ and $b$ are distinct and non-zero, the Hessian of $(\nabla g)^{T}(R \nabla f)$ will be non-degenerate, thus proving that the level set $(\nabla g)^{T}(R \nabla f)=0$ will have valence four at the origin.

We note that requiring $f$ to be a Morse function is not sufficient to satisfy Theorem 4.3, however any Morse function can be perturbed such that it remains Morse and meets the requirements in the theorem [15]. For example, consider the function $f=x^{2} y-x^{2}-y^{2}$, shown in Figure 3. This function is Morse, but has non-distinct eigenvalues at the origin and produces a valence six point for the Jacobi set $\mathbb{J}\left(f,\|\nabla f\|^{2}\right)$. As seen in Figure 3(b-d), the function can be perturbed to satisfy Theorem 4.3, which creates a valence four critical point and a birth-death point.

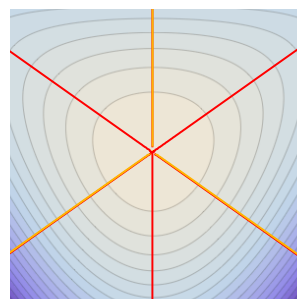

(a)

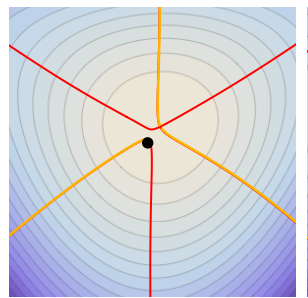

(b)

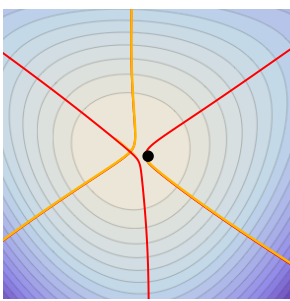

(c)

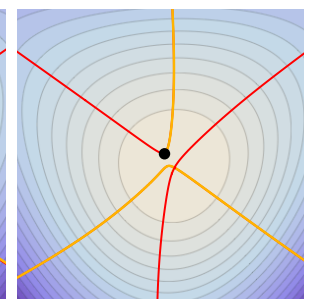

(d)

Figure 3: The contours of $f=x^{2} y-x^{2}-y^{2}$ and its ridge-valley graph as computed and plotted by Mathematica. Ridges (red) and pseudo-ridges (orange) are seen crossing at the maxima. (a) The maxima in $f$ has a valence six structure. (b)(c)(d) When perturbed the maxima becomes valence four with a birth-death point located nearby. 
Combining the discussion above we define the Ridge-Valley (RV) graph as the Jacobi set of $f$ and $\|\nabla f\|^{2}$ and can derive several interesting global properties:

Definition 4.4 (Ridge-Valley Graph). Given a Morse function $f$ such that at all critical points of $f$ the Hessian have distinct, non-zero eigenvalues, the non-generic Jacobi set $\mathbb{J}\left(f,\|\nabla f\|^{2}\right)$ is called the Ridge-Valley graph of $f$.

The RV graph of $f$ consists of a collection of arcs and nodes such that:

1. Arcs are open, smoothly embedded 1-manifolds;

2. Nodes are the critical points of $f$ which have valence four and points at which the Jacobi classification switches which have valence two;

3. Arcs have a consistent classification as (pseudo-)ridge or (pseudo-)valley;

4. At maxima/minima of $f$ pseudo-ridges/-valleys cross ridges/valleys; and

5. At saddles of $f$ ridges and valleys cross.

Property (1) and (2) follow directly from the definition of Jacobi sets and Theorem 4.3. Similarly, (3) is a consequence of the classification of Jacobi points. For (4) we note that certainly the level set curvature in an $\epsilon$ neighborhood of a maximum is positive and thus all incident arcs are classified ridge or pseudoridge. Since, on the level set around a maximum of $f, g$ must alternate between maxima and minima property (4) follows. Similarly, for (5), the well-known level set structure around a saddle guarantees four lines alternating valley- and ridge-types (positive and negative level set curvature). Furthermore, as the ridges/valleys approach the saddle the gradient magnitude approaches 0 which guarantees a minimum of $g$ along the level set from which property (5) follows.

Similar to the ridge-valley-connector-curves of [15] for height ridges, the RV graph provides insight into the global structure of Jacobi ridges and valleys. Confirming the human intuition, ridges of two-dimensional functions are lines and are pair-wise disjunct. The second property combined with the valence four structure around critical point has an important non-intuitive consequence: Even at local maxima of $f$ ridges cannot merge. Intuitively, one might expect multiple ridges to be incident to the same maximum just as mountain crests (the typical mental image for ridges) can converge to a single peak. According to Theorem 4.3 this is generally not possible for ridges of Morse functions. This insight reveals a fundamental problem with ridge detection algorithms that classify individually pixels. By classifying single points as ridge/non-ridge one must, on the one hand create unbroken lines suggesting a loose classification, on the other hand one is not allowed to create any non-valence two structures suggesting a conservative classification. Deciding the correct connections is a global problem difficult to solve with local heuristics.

\section{Algorithm}

In this section we will describe how the concepts for smooth functions discussed above can be applied to sampled functions. First, we will use the connection to Jacobi sets to create a new combinatorial algorithm to extract the 
set of Jacobi points from piecewise linear functions. Second, we show how to extend the traditional algorithms for Jacobi set extraction to handle non-generic functions in order to construct a provably consistent Ridge-Valley graph.

\subsection{Jacobi Sets on Piecewise Linear functions}

Edelsbrunner and Harer [21], using the concept of Lagrangian Multipliers, show that the points of the Jacobi set are equivalent to the points where the gradients of $f$ and $g$ are linearly dependent $(\nabla g+\lambda \nabla f=0$ or $\nabla f+\lambda \nabla g=0)$. Defining $h_{\lambda}=g+\lambda f$ with $\nabla h_{\lambda}=\nabla g+\lambda \nabla f$ leads to an alternative definition of the Jacobi set:

Lemma 5.1 (Jacobi Set). The Jacobi set $\mathbb{J}(f, g)$ of two generic Morse functions $f$ and $g$ is equivalent to the set

$$
\begin{aligned}
\mathbb{J}(f, g)= & \{x \in \mathbb{M} \mid \exists \lambda \in \mathbb{R} \text { s.t. } x \text { is a critical point of } \\
& \left.h_{\lambda}=g+\lambda f, \text { or } x \text { is a critical point of } f\right\}
\end{aligned}
$$

With a slight abuse of notation one can assume $h_{ \pm \infty}= \pm f$ and define the Jacobi set as the set of critical points of the one-parameter family of functions defined by $h$. Alternatively, this set can be described as the paths the critical points of $h_{\lambda}$ take as $\lambda$ is swept from $-\infty$ to $\infty$. This reduces the computation of Jacobi sets to the detection of critical points albeit for an infinite number of functions.

Clearly, if $f$ and $g$ are piece-wise linear so is $h$. Following the standard approach $[28,29]$, critical points of a piece-wise linear function can be defined by classifying their neighborhoods. Consider a triangulation $T$ that is a collection of vertices $V$, edges $E$, and faces $F$, with the face relation. Function values are available at vertices, and are linearly interpolated on the edges and faces to recover a continuous function. The star of a vertex $v$ is defined as the collection of all simplices containing $v$, and the link of $v$ is the boundary of the closure of the star of $v$. The upper/lower link of $v$ is the portion of the link with function values above/below $v$. A vertex is determined to be critical by the sum of the components of the upper and lower link. For example a regular (non-critical) vertex has one component of the upper and lower link, Figure 4(a). A maximum (or minimum) simply has one component of lower link (or upper link), Figures 4(b) and 4(c). A saddle point of order $n$ has $n+1$ components of both the upper and lower link, Figure 4(d) shows a saddle of order 1.

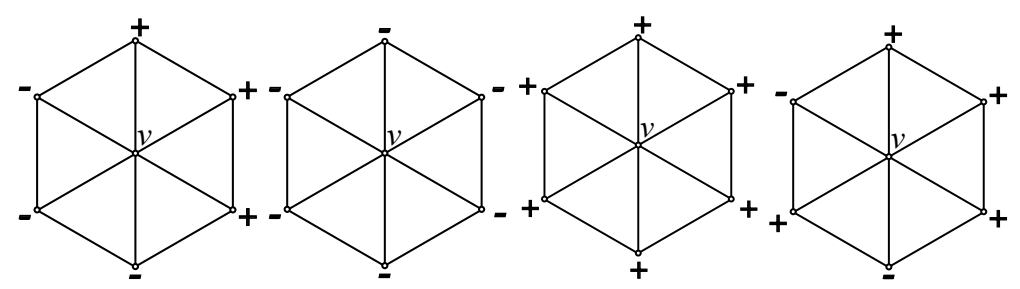

(a) Regular

(b) Maximum

(c) Minimum

(d) Saddle

Figure 4: Examples of the classification of regular and critical points for a piecewise linear function. The vertices neighboring $v$ are marked $+/-$ if they are higher or lower than $v$. 
Given a piece-wise linear $h_{\lambda}$ and assuming for now that neighboring vertices have distinct function values the critical points of $h_{\lambda}$ can therefore be detected using simple vertex comparisons. Furthermore, as $\lambda$ is swept from $-\infty$ to $\infty$ critical points can only change as edges flip direction from ascending to descending or vice versa. Given an edge $e=\overline{u v}$ its direction will flip at $\lambda_{e}=(g(u)-g(v)) /(f(v)-f(u))$. Each edge will flip exactly once and using simulation of differentiability [21] we can assume these flips are ordered and occur at distinct $\lambda$ values.

What remains is to determine whether an edge flip affects any critical points. Referring to Figure 5 only $u$ and $v$ can be affected by flipping $\overline{u v}$ and the number of components of their upper and lower link change only if $a$ and $b$ are either both in the upper or both in the lower link of $u$ and $v$ at the time of the flip. Intuitively, if an edge flip changes the critical points of $h_{\lambda}$ than before the split either $u$ or $v$ (or both) contain a critical point which during the split instantaneously "moves" across the edge. As discussed in [21], one can therefore examine each edge individually and determine whether it is part of the Jacobi set or not. This collection of edges is then "unfolded" in a post-processing step to extract non-intersecting one-manifolds from the collection of edges. Here we use a slight modification of this algorithm proposed in [25] which preserves additional invariants and produces qualitatively better results.

\subsection{Combinatorial Ridge-Valley Graphs}

As discussed in Section 4, the ridge-valley graph is a degenerate Jacobi set of $f$ and $g=\|\nabla f\|^{2}$. We use the piecewise linear formulation of Jacobi sets to compute the ridge-valley graph for a piece-wise linear function $f$ and $g$, where $g$ is constructed by any of the existing gradient estimation schemes. For example, many computer simulations internally express $f$ using higher order interpolations techniques and $g$ can be evaluated from these interpolations.

Both techniques discussed above will extract a set of 1-manifolds as they are designed for Jacobi sets of generic Morse functions. The RV graph is a degenerate Jacobi set and has a more varied structure as discussed in Section 4. Thus, we modify the techniques in order to ensure the proper structure.

Additionally, in the Jacobi set computation $f$ and $g$ are both considered to be piecewise linear, which can potentially lead to inconsistencies between the relationship of $f$ and $g$, specifically at the critical points of $f$. Thus the value of $g$ and the level set curvature are set explicitly at critical points of $f$ and the valence four criteria is enforced, when not met. The following subsections describe how these inconsistencies are remedied in order to preserve the correct structure of the RV Graph.

\subsubsection{Co-locating Critical Points}

Approximating the gradient magnitude numerically does not guarantee to produce minima of $g$ at all critical points of $f$. A first order adjustment is to set $g=0$ for all critical points of $f$. However, even enforcing $g>0$ for all non-critical vertices of $f$ using, for example, symbolic perturbation does not yet 
guarantee the desired structure. There can exist edges of $T$ connecting critical points of $f$. Since, two minima of $g$ cannot be connected by an edge we choose to split the edge and introduce a new vertex separating the two critical points. Thus, we guarantee that all critical points of $f$ are also minima of $g$.

\subsubsection{Edge Classification}

The detection of Jacobi points is equivalent to finding edges in the Jacobi set of $f$ and $g=\|\nabla f\|^{2}$. However, the classification of edges as (pseudo)ridges/valleys as described in Definition 3.2 remains to be determined. As shown in Table 2, there are two criteria to classify a Jacobi point $x$ : whether $g(x)$ is a maximum or minimum along a level set of $f$, and whether $f(x)$ is a maximum or minimum tangent to the level set.

Conveniently, the first criterion is handled implicitly during the computation of the Jacobi set. Consider the edge $\overline{u v}$ shown in Figure 5 and let $L(a)$ be the level set of one of its neighbors. If $\overline{u v}$ is parametrized from $t \in(0,1)$ then $L(a)$ intersects at $t=\frac{f(a)-f(u)}{f(v)-f(u)}$. Comparing the value of $g$ at the intersection to the value of $g$ at the vertex $a$ leads to:

$$
\begin{array}{ccl} 
& g(t)=g(u)+(g(v)-g(u)) t & <g(a) \\
\Leftrightarrow & g(u)-g(a)+(g(v)-g(u)) \frac{f(a)-f(u)}{f(v)-f(u)} & <0 \\
\Leftrightarrow & g(u)-g(a)+\lambda_{u v}(f(u)-f(a)) & <0 \\
\Leftrightarrow & g(u)+\lambda_{u v} f(u)-\left(g(a)+\lambda_{u v} f(a)\right. & <0 \\
\Leftrightarrow & h_{\lambda_{u v}}(u) & <h_{\lambda_{u v}}(a)
\end{array}
$$

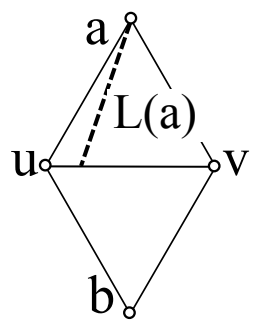

Figure 5: An edge $\overline{u v}$ of $T$ and the level set, $L(a)$, that goes through vertex $a$.

Note that, $L(a)$ might intersect the line outside of $\overline{u v}$. Never-

theless, since $f$ and $g$ are linear on the triangle $u v a$, the comparison remains valid as there is no restrictions on the value of $t$. Thus if $h_{\lambda_{u v}}(a), h_{\lambda_{u v}}(b)>h_{\lambda_{u v}}(u)$, then $u v$ is a minimum of $g$ on level sets of $f$ and is classified as non-pseudo. If $h_{\lambda_{u v}}(a), h_{\lambda_{u v}}(b)<h_{\lambda_{u v}}(u)$, then $g$ is a maximum and $u v$ is classified as pseudo.

As discussed in Section 4, whether $f$ has a maximum or minimum tangent to the level set is equivalent to determining the sign of the level set curvature, (Eqn. (1)). Using any existing curvature estimation techniques each vertex in the triangulation $T$ is given a value for level set curvature and the curvature of each edge is defined as the average of the values of its vertices. Depending on the sign each edge is classified as ridge or valley.

From Equation (1) it is clear that for critical points of $f$, the level set curvature is undefined. Level set curvature approaches infinity as one approaches a maximum and negative infinity for a minimum. At a saddle, level set curvature will approach infinity or negative infinity depending on whether the saddle is approached from above or below. In order to maintain consistency, the values of the level set curvature at critical points of $f$ are assigned $\pm \infty$ with the sign on the saddle dependent upon the approach. With these values specifically assigned, all edges bordering a maximum (minimum) will have positive (negative) curvature giving all edges a ridge (valley) classification. An edge of a 
saddle point will be give a ridge/valley classification if the edge's other vertex is higher/lower than the saddle point.

\subsubsection{Valence correction}

The unfolding processes in $[21,25]$ will create a set of non-intersecting loops from the Jacobi edges. However, as discussed above, the RV graph of a Morse function contains loop intersections at critical points of $f$ indicated by valence four nodes. Thus at critical points of $f$ we must modify the unfolding process to establish the structure of the RV graph.

The cases of extrema and saddles are handled separately. For each extrema $p$ of a piecewise linear $f$, there exist three cases: (i) $p$ is incident to four; (ii) more than four; or (iii) two Jacobi edges. In case (i) no correction is needed. For case (ii) we note that in [21] it is shown that $p$ must have an even valence. Thus, as shown in Figure 3 for the smooth case, any perturbation of $f$ will split a higher valence extremum into a valence four plus additional birth-death points. We emulate this perturbation by pairing adjacent edges, considering them no longer incident to $p$, until only four unpaired edges remain which form the proper valence four extremum. To correct case (iii) we note that there exist two possible explanation for a valence two extremum. First, we know that ridges and pseudo-ridges can form a loop, see Figure 1(a). In a sampled function this loop might be too small to appear in the mesh and thus the extremum is left with only two Jacobi edges. Second, due to numerical instabilities, for example, in the gradient estimation the birth-death point that in the smooth case would have joined the co-located critical points of $f$ and $g$ (see Figure 2) may actually not be co-located. As a result, we find the structure of Figure 6 with a critical point of $f$ with valence two close to a birth-death point. To decide between the two scenarios we use Algorithm 1.

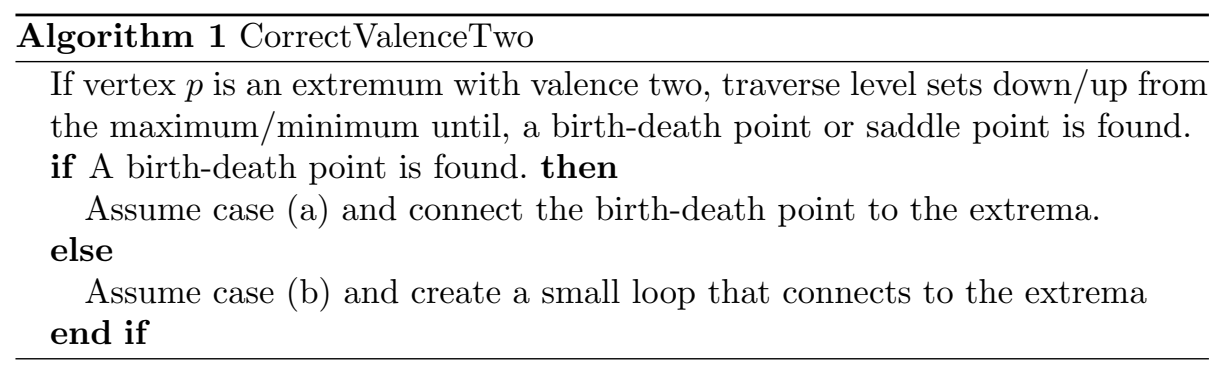

By limiting the search for a birth-death point to the level sets around an extremum until a saddle is found, we are guaranteed that two extrema cannot search and find the same birth-death point to connect to itself, as the level set of the saddle isolates the extrema from any other critical points. Assuming an invisible loop for every valence two extremum would create a correct structure. However, we generally find the search for birth-death points to produce a more intuitive structure. Figure 6 shows extrema that are valence two with birthdeath points nearby. With Algorithm 1, these examples would have the nearby birth-death point merged with the extrema. 


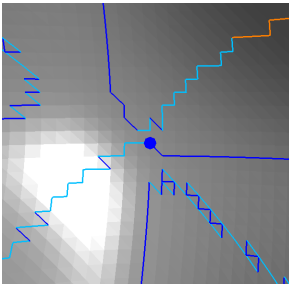

(a)

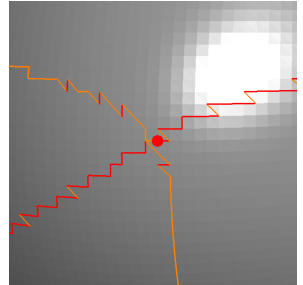

(b)

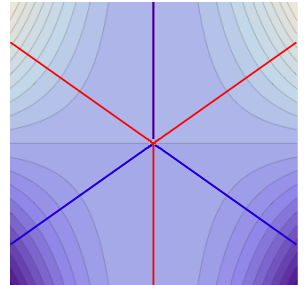

(c)

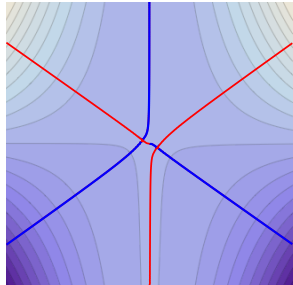

(d)

Figure 6: (a) A valence two minimum (blue) with multiple birth-death points nearby. (b) A valence two maximum (red) with a birth-death point just to the left of the maximum. (c) A valence six monkey saddle changes into two simple saddles (d) after perturbation.

Saddle points of $f$ are handled slightly differently than extrema. Due to the co-location of critical points, the detection of Jacobi edges guarantees that all non-degenerate saddle points of $f$ will have at least valence four, see Figure 7 . During the $\lambda$ sweep, if $v$ is a saddle point of $f$ then it has two components of lower link at $\lambda=-\infty$. The vertex $v$ is also a minimum of $g$ thus at $\lambda=0$, the vertex will be a minimum and have only one component of the upper link. Thus in the interval $\lambda \in(-\infty, 0)$ both components of the lower link must have collapsed and in doing so created at least two edges in the Jacobi set. During the interval $\lambda \in(0, \infty)$, two lower links must be created returning the vertex to a saddle at $\lambda=\infty$. Again this creates at least two edges in the Jacobi set, thus guaranteeing valence of at least four for saddle points. From section 5.2.2 we know that when a lower link is collapsed or created the corresponding edge will be minimal in $g$ along the level set of $f$ and thus is classified nonpseudo. Furthermore, the well known level set curvature structure around a saddle guarantees four alternating ridge and valley edges. The same results can be extrapolated for higher order saddle points of $f$, where a valence of at least $2 n+2$ minimal edge is assured for saddles of order $n$.

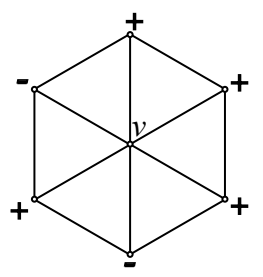

(a) $\lambda=-\infty$

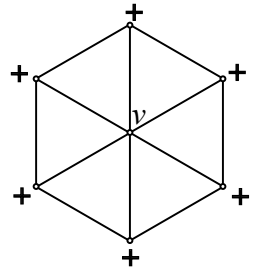

(b) $\lambda=0$

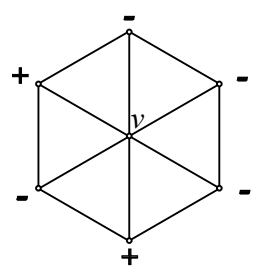

(c) $\lambda=\infty$

Figure 7: Saddles must have at least valence four. (a) At $\lambda=-\infty$ there exist two components of the lower link. (b) At $\lambda=0$, both lower link components have collapsed marking two edges as in the Jacobi set. (c) At $\lambda=\infty$ there are again two lower link components, that mark at least two additional edges as in the Jacobi set upon their creation. Thus $v$ is incident to at least four Jacobi edges.

It is possible for a saddle to have more than its proper number of Jacobi edges. If during the $\lambda$-sweep a lower link is split before it collapses, then there 
will be additional edges. From Section 5.2.2 the edge flip that split the lower link will create a maximal edge. As is done with an extrema of higher valence, this edge is paired with either of its adjacent minimal edges, is disconnected from the saddle, and is considered incident to a birth-death point.

Piecewise linear functions can support higher order saddle points. A saddle points of order $n$ can be split into $n$ saddles of order 1 . Figure 6 , shows the ridge-valley graph for the classic monkey saddle $f=x^{2} y$ and the resulting ridge-valley graph when perturbed. The saddle point has been split into two separate saddle points connected by the ridge-valley graph creating two valence four critical points.

\subsection{Consistency of Computed RV Graphs}

Definition 4.4 lists several properties of the Ridge-Valley graph for extended Morse functions. We will show that the Ridge-Valley graph detected on a piecewise linear $f$ using the above algorithm will satisfy the same properties.

1. Arcs are open, smoothly embedded 1-manifolds. The unfolding techniques of $[21,25]$ will extract 1-manifolds from the edges of the Jacobi set, except at critical points.

2. Nodes are the critical points of $f$ which have valence four and points at which the Jacobi classification switches which have valence two. From the section above, the critical points of $f$ will have valence four or will be corrected to valence four. All other points will have valence two from the unfolding.

3. Arcs have a consistent classification as (pseudo-)ridge or (pseudo-)valley. From section 5.2.2 each edge has one classification. Arcs for the piecewise linear RV graph are formed by sequences of edges of the same classification and thus by definition have consistent classification.

4. At maxima/minima of $f$ pseudo-ridges/-valleys cross ridges/valleys. All extrema of $f$ have valence four, and thus must have two maximal and two minimal edges that alternate around the extrema. The level set curvature at maxima has been set to $\infty$, thus all edges incident to a maximum have positive level set curvature and are either pseudo-ridge or ridge. Thus a maximum has two ridge and two pseudo-ridge edges that alternate around the maximum or "cross". Similarly, pseudo-valleys and valleys cross at minima.

5. At saddles of $f$ ridges and valleys cross. Saddles are incident to four minimal edges. The way level set curvature is assigned to saddles, the two edges in the upper link of the saddle will be ridge, and the two edges in the lower link will be valley. Thus valleys and ridges cross at saddle points.

\section{Discussion}

The concept of the Ridge-Valley graph as a Jacobi set and the presented algorithm for its computation has several advantages for practical applications. 
While the classification of all the edges in the RV graph needs level set curvature, which requires a second derivative, the actual geometry of the RV graph is computed using only the magnitude of the gradient, a first order quantity. Using just an estimate of the gradient magnitude the RV graph can be computed combinatorially in a provable consistent manner. As such the RV graph computation does not rely on numerical calculations and is more robust compared to the detection of height ridges, which requires second order derivatives and eigenvalue/vector computations.

\subsection{Length of Scale and Topological Simplification}

Currently the methods for examining height ridges at different lengths of scale and filtering out noise involves passing the data through a low-pass filter [2]. A potential advantages of expressing the Ridge-Valley graph as a discrete graph structure is that this could allow a structural simplification to filter out noise and create a multi-resolution representation of ridges and valleys similar to the work done in the Morse-Smale complex [30, 31, 32]. Such an approach could potentially allow for a more adaptable approach, such as local filtering, as opposed to global and the ability to remove features based on user-defined criteria. Currently techniques into Jacobi set simplification [33] remain problematic in general and furthermore, are not able to handle non-generic Jacobi sets. Further investigation on whether topological simplification can give a suitable length of scale analysis is needed before applying the presented algorithm to analytical purposes.

Without a topological simplification our current technique is subject to sampling and mesh artifacts, as well as noise in the data and small scale features. Nevertheless, unlike other techniques, the RV graph is guaranteed to be consistent with the known mathematical structure and all ridges and valleys are guaranteed to be 1-manifolds.

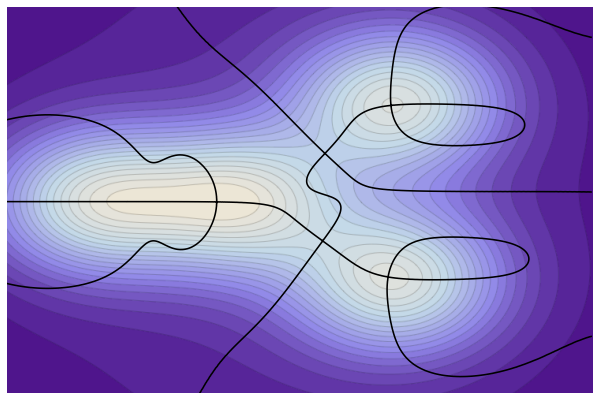

(a)

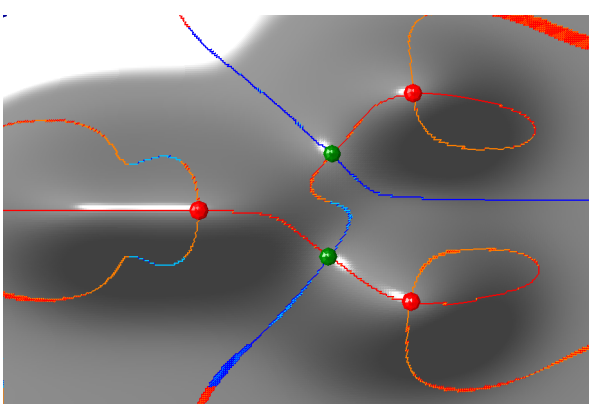

(b)

Figure 8: Comparison between the analytical and computed ridge-valley graph of $f=$ $\exp \left[-\left(8(x+0.4)^{2}+4 y^{2}\right)\right]+\exp \left[-8(x-.5)^{2}-4 y^{2}\right]+\exp \left[-8(x-0)^{2}-4(y-.77)^{2}\right]+\exp [-8(x-$ $\left.0)^{2}-4(y-1.5)^{2}\right]+0.2 \exp \left[-0.3 x^{2}-0.3(y-.5)^{2}\right]$. (a) The contours of $f$ and its ridge-valley graph are computed and plotted in Mathematica. (b) The function $f$ is sampled on a $301 \times 401$ resolution regular triangle mesh and the ridge-valley graph is computed using the described algorithm. 


\section{Results}

This section shows some example RV graphs for both analytic and practical data sets. The first example shows an analytical function where the human intuition would expect two ridges to merge. Figure 8(a) shows the contour map and the level set $(\nabla f)^{T} H(f)(R \nabla f)=0$ as computed by Mathematica. Figure 8(b) shows the computed RV Graph. As one can see in both figures, the ridges going through the maxima on the right do not merge into a single ridge, but one of them changes classification and connects into a saddle point instead. As expected, the combinatorial extraction creates some small artifacts especially in regions of near zero gradients.

Next we examine an artificial terrain created by superimposing multiple Gaussian distributions. Figure 9 shows the terrain, the full RV graph, the ridges and the valleys of the terrain. While overall the features extracted from the RV graph appear to correspond well with human perception, there again exist small artifacts due to sampling and meshing artifacts.

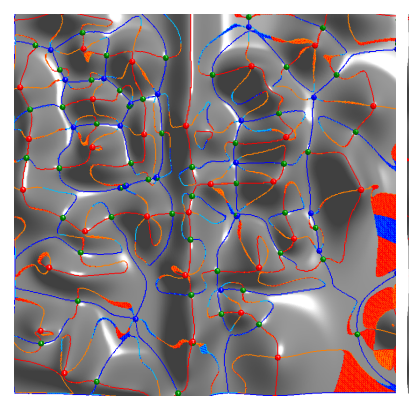

(a)

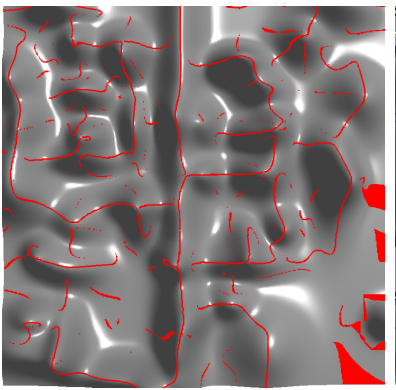

(b)

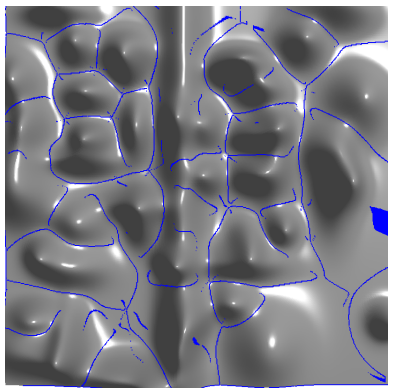

(c)

Figure 9: An artificial terrain constructed by superimposing random Gaussian distributions. (a) The computed RV graph with maxima (red dots), minima (blue dots), and saddle point (green dots) connected by arcs of ridge (red), valley (blue), pseudo-ridge (orange) and pseudovalley (teal). (b) The ridges of the terrain. (c) The valleys of the terrain.

For an example of the ridge detection on simulation data, we examine a Finite Time Lyapunov Exponent (FTLE) field where ridges of the field are considered Lagrangian Coherent Structures (LCS) [34, 35]. These LCS represent material boundaries which have near-zero cross flow in the fluid. Figure 10 shows the ridges of the RV graph for the FTLE field of fluid flow around a cylinder and the ridges found in the RV graph. While the large scale ridges are readily found, many small scale ridges are also detected.

\section{Future Work}

While these examples show encouraging results, there remain several critical issues that need to be resolved before the presented algorithm is suitable for analytical purposes. The most pressing issue is addressing the need for a length of scale analysis through topological simplification as discussed in Section 6.1. Many analyses require such capabilities and as seen in the previous section, the 


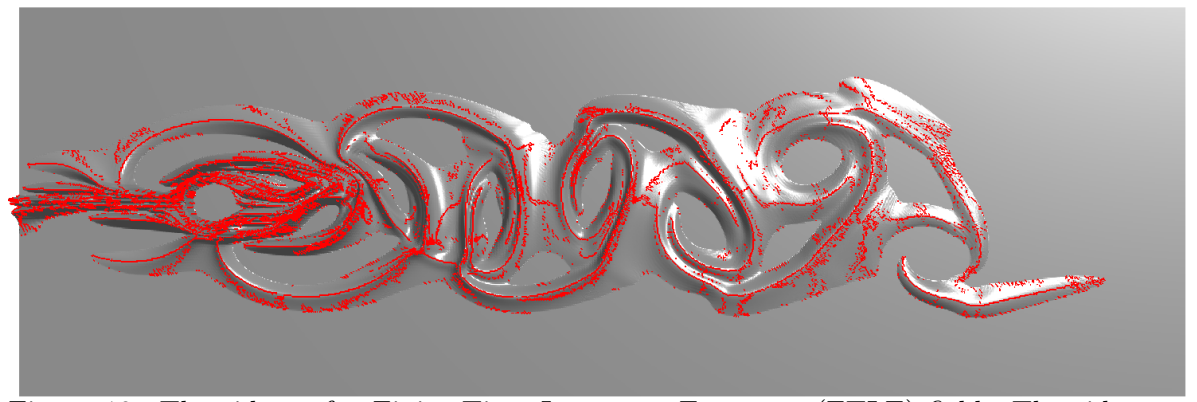

Figure 10: The ridges of a Finite Time Lyapunov Exponent (FTLE) field. The ridges are shown in red. While the large scale ridges are captured, many small scale ridges and noise are also present in the RV graph, showing a need for topological simplification.

algorithm currently detects many small scale ridges and valleys, often on the order of a single triangle.

Other minor issues arise from considering the functions $f$ and $g$ as piecewise linear, unlike many of the current methods, which use higher order interpolation or fitting. The structure of the RV graph is now dependent upon the meshing procedure used. Ideally, this will be a minor issue that is rendered insignificant by a proper simplification scheme, but is currently an open question. The piecewise linear structure will also produce jagged ridge lines as all ridges are found along edges of the mesh, which results in visually unappealing ridges. However this is likely easily remedied by applying some constrained smoothing procedure on the lines.

Much of the current work on ridge detection involves finding two-dimensional ridge surfaces in three-dimensional data. It is not clear that the Jacobi set definition of ridge is easily extendible to additional dimensions, unlike the height ridge definition. Jacobi sets themselves are always one-dimensional, regardless of the dimension in which the are embedded, so the theory will need to be generalized in order to extract two-dimensional ridge surfaces.

\section{Summary}

We have examined Jacobi ridges, a definition of ridges that is closely related to the traditional height ridge definition, but remains invariant under monotone transformation. Furthermore, we have derived the Ridge-Valley graph to describe the global structure of Jacobi ridges for Morse functions with simple Hessians. Finally, we show how ridges can be expressed as non-generic Jacobi sets and use this insight to develop the first combinatorial algorithm to extract a provably consistent RV graph from piecewise linear data. The RV graph provides insights into the global nature of ridges and the connection to Jacobi sets promises a new framework to compute and analyze ridges. The theory of Jacobi sets shows the potential to enable the structurally correct simplification of the RV graph without globally smoothing the data and potentially the creation of a hierarchical RV graph in the tradition of hierarchical Morse-Smale complexes. 
While the presented algorithm is not currently suitable for state of the art analysis, its combinatorial approach shows much promise for robust computation if its current limitations are overcome.

Overall the Ridge-Valley graph opens the possibility for a greater understanding of the global structure of the ridges and valleys of a Morse function and presents the opportunity to increase the robustness of ridge detection by utilizing combinatorial algorithms that take advantage of its Jacobi set structure.

\section{References}

[1] J. Gauch, S. Pizer, Multiresolution analysis of ridges and valleys in greyscale images, Pattern Analysis and Machine Intelligence, IEEE Transactions on 15 (6) (1993) $635-646$.

[2] T. Lindeberg, Edge detection and ridge detection with automatic scale selection, International Journal of Computer Vision 30 (1996) 465-470.

[3] D. Eberly, R. Gardner, B. Morse, S. Pizer, C. Scharlach, Ridges for image analysis, Jounral of Mathematical Imaging and Vision 4 (1994) 353-373.

[4] T.Schultz, H. Theisel, H.-P. Seidel, Crease surfaces: From theory to extraction and application to diffusion tensor mri, IEEE Transactions on Visualization and Computer Graphics 16 (2010) 109-119.

[5] X. Tricoche, G. Kindlmann, C.-F. Westin, Invariant crease lines for topological and structural analysis of tensor fields, IEEE Transactions on Visualization and Computer Graphics 14 (2008) 1627-1634.

[6] J. Frank, S. Kaiser, High-resolution imaging of dissipative structures in a turbulent jet flame with laser rayleigh scattering, Experiments in Fluids 44 (2008) 221-233.

[7] De Saint-Venant, Surface a plus grande pente constituees sur des lignes courbes, Bulletin de las soc. philomath. de Paris.

[8] J. Boussinesq, Sur une propriété remarquable des points où les lignes de plus grande pente d'une surface ont leurs plans osculateurs verticaux, et sur la différence qui existe généralement, à la surface de la terre, entre les lignes de faite et de thalweg et celles le long desquelles la pente du sol est un minimum, C.R. Paris 73 (1871) 1368.

[9] J. Boussinesq, Cours d'analyse infinitesimal I, Vol. 2, Paris, 1887.

[10] P. Breton de Champ, Memoire sur les lignes de faite et de thalweg que l'on est conduit a considerer en topographie, Journal de Mathematique Pure et Appliquees 3 (1877) 99-114.

[11] C. Jordan, Sur les lignes de faite et de thalweg, CR Acad. Sc. Paris 74 (1872) 1459-1475. 
[12] R. Rothe, Zum pronelm des talwegs, Sitz.ber. d. Berliner Math. Gesellshaft 14 (1915) 51-69.

[13] J. J. Koenderink, A. J. van Doorn, Local features of smooth shapers: Ridges and courses, Geometric Methods in Computer Vision II 2031 (1993) 2-13.

[14] R. Haralick, Ridges and valleys in digital images, CVGIP 22 (1983) 28-38.

[15] J. Damon, Generic structure of two-dimensional images under Gaussian blurring, SIAM Journal on Applied Mathematics 59 (1) (1998) 97-138.

[16] D. Eberly, Ridges in Image and Data Analysis, Kluwer Academic Publishers, 1996.

[17] R. Peikert, M. Roth, The 'parallel vectors' operator: a vector field visualization primitive, in: Proc. IEEE Visualization '99, IEEE Computer Society Press, 1999, pp. 263-270.

[18] J. Furst, S. M. Pizer, Marching ridges, in: IASTED International Conference on Signal and Image Processing., 2001.

[19] R. Peikert, F. Sadlo, Height Ridge Computation and Filtering for Visualization, in: Proceedings of Pacific Vis 2008, 2008, pp. 119-126.

[20] G. Kindlmann, X. Tricoche, C.-F. Westin, Delineating white matter structure in diffusion tensor mri with anisotropy creases, Medical image analysis 11 (5) (2007) 492-502.

[21] H. Edelsbrunner, J. Harer, Jacobi sets of multiple morse functions, Foundations of Computational Mathematics (2004) 37-57.

[22] H. Edelsbrunner, J. Harer, A. Mascarenhas, V. Pascucci, J. Snoeyink, Time-varying reeb graphs for continuous space-time data, Computational Geometry 41 (3) (2008) 149 - 166.

[23] H. Edelsbrunner, J. Harer, V. Natarajan, V. Pascucci, Local and global comparison of continuous functions, in: In "Proc. IEEE Conf. Visualization, IEEE Computer Society, 2004, pp. 275-280.

[24] C. Luo, I. Safa, Y. Wang, Approximating gradients for meshes and point clouds via diffusion metric, in: Proc. of the Symposium on Geometry Processing, 2009, pp. 1497-1508.

[25] G. Norgard, A. Gyulassy, V. Pascucci, P.-T. Bremer, Extending jacobi set theory: Alignment and robust unfolding, submitted (2011).

[26] G. Kindlmann, X. Tricoche, C.-F. Westin, Anisotropy creases delineate white matter structure in diffusion tensor mri, in: Medical Image Computing and Computer-Assisted Intervention - MICCAI 2006, Vol. 4190, 2006, pp. $126-133$. 
[27] F. Sadlo, R. Peikert, Efficient visualization of lagrangian coherent structures by filtered amr ridge extraction, IEEE Transactions on Visualization and Computer Graphics 13 (6) (2007) 1456 -1463.

[28] T. F. Banchoff, Critical points and curvature for embedded polyhedral surfaces, Differential Geometry 3 (1) (1967) 257-268.

[29] H. Edelsbrunner, J. Harer, A. Zomorodian, Hierarchical Morse-Smale complexes for piecewise linear 2-manifolds, Discrete Comput. Geom. 30 (2003) $87-107$.

[30] P.-T. Bremer, B. Hamann, H. Edelsbrunner, V. Pascucci, A topological hierarchy for functions on triangulated surfaces, IEEE Transactions on Visualization and Computer Graphics 10 (4) (2004) 385-396.

[31] P.-T. Bremer, Topology-based multi-resolution hierarchies, Ph.D. thesis, University of California, Davis, Davis, CA (2004).

[32] A. Gyulassy, L. G. Nonato, P.-T. Bremer, C. Silva, V. Pascucci, Robust topology-based multiscale analysis of scientific data, Computing in Science and Engineering 11 (5) (2009) 88-95.

[33] N. Suthambhara, V. Natarajan, Simplification of jacobi sets, in Topological Data Analysis and Visualization: Theory, Algorithms and Applications. Springer-Verlag, Mathematics and Visualization Series, 2010, to appear.

[34] G. Haller, G. Yuan, Lagrangian coherent structures and mixing in twodimensional turbulence, Physica D: Nonlinear Phenomena 147 (3-4) (2000) $352-370$.

[35] S. C. Shadden, F. Lekien, J. E. Marsden, Definition and properties of lagrangian coherent structures from finite-time lyapunov exponents in twodimensional aperiodic flows, Physica D: Nonlinear Phenomena $212(3-4)$ (2005) $271-304$. 